\title{
Theranosties
}

Editorial

2013; 3(5):354-355. doi: 10.7150/thno.6480

\section{Theranostic Upconversion Nanoparticles (II)}

\author{
Gang $\operatorname{Han}^{1}$ and Guanying Chen ${ }^{\circledR}$ \\ 1. Department of Biochemistry and Molecular Pharmacology, University of Massachusetts Medical School, Worcester, Massachusetts \\ 01605 (USA) \\ 2. Institute for Lasers, Photonics, and Biophotonics, University at Buffalo, State University of New York, Buffalo, New York 14260 (USA)
}

$\square$ Corresponding author: guanying@buffalo.edu (Guanying Chen) and Gang.Han@umassmed.edu (Gang Han).

(c) Ivyspring International Publisher. This is an open-access article distributed under the terms of the Creative Commons License (http://creativecommons.org/ licenses/by-nc-nd/3.0/). Reproduction is permitted for personal, noncommercial use, provided that the article is in whole, unmodified, and properly cited.

Received: 2013.04.15; Accepted: 2013.04.15; Published: 2013.04.25

\begin{abstract}
This theme issue provides a comprehensive collection of original research articles as well as reviews on the creation of diverse types of theranostic upconversion nanoparticles, their fundamental interactions in biology, as well as their biophotonic applications in noninvasive diagnostics and therapy.
\end{abstract}

Key words: Upconversion, Bioimaging, Nontoxicity, Plasmonics, Multimodal.

This issue (volume 3, number 5) is the second theme issue in the series of "Theranostic Upconversion Nanoparticles". The articles in this theme issue encompass 2 original research articles and 3 review articles. Yang et al. reviewed recent investigations on using upconversion nanoparticles (UCNPs) for the detection of biomolecules (avidin, ATP, etc.), ions (cyanide, mercury, etc.), small gas molecules (oxygen, carbon dioxide, ammonia, etc.), as well as for in vitro temperature sensing [1]. Liu et al. summarized the latest progresses regarding the applications of upconversion nanoparticles for photodynamic therapy, near-infrared (NIR) laser triggered drug and gene delivery, as well as several other upconversion nanoparticle-based cancer therapeutic approaches [2]. Zhang et al. reviewed recent design and fabrication of multifunctional upconversion-magnetic hybrid nanostructured materials as well as their use for enhanced bioimaging and controlled drug delivery [3]. He et al. utilized the evanescent wave (EW) for the excitation of upconverison nanoparticles, providing a scanning-free as well as high contrast and high spatiotemporal resolution method of tracking single nanoparticles [4]. Li et al. reported on the use of upconversion $\mathrm{NaLuF}_{4}: \mathrm{Yb}$, Tm nanophosphors for real-time upconversion luminescence imaging under ambient light and high-resolution X-ray CT imaging [5]. In sum, this special issue presents the frontline theranostic applications of upconversion nanoparticles, which in turn encourages new directions for combined diagnosis and therapy.

Despite recent exciting developments in theranostic upconversion nanoparticles, challenges remain for those in the field. In this regard, we asked the following two questions: What is the major hurdle in the development of upconversion nanoparticles for biomedical applications? What may be the next big thing in his area? In order to answer these questions, we surveyed some of the leading scientists in the field. In the previous issue (volume 3, number 4) of Theranostics, we have presented opinions of several leading scientists in this field. In the subsequent paragraph, we continue to present the consensus of other ten leading scientists in five countries from this survey.

So, what is the major hurdle in the development of upconversion nanoparticles for biomedical applications?

Prof. Xiaogang Liu from Singapore National University, Prof. Xueyuan Chen from Fujian Institute of Research on the Structure of Matter, Chinese 
Academy of Sciences, Prof. Stefan Andersson-Engels from Lund University, Prof. Zhuang Liu from Soochow University, Prof. Fan Zhang from Fudan University, Prof. Jun Lin from Changchun Institute of Applied Chemistry, Chinese Academy of Sciences, Prof. Sailing He from Zhejiang University \& Royal Institute of Technology (Sweden), Prof. Hong Zhang from University of Amsterdam, Prof. Xuefeng Yu from Wuhan University and one scientist from Molecular Foundry, Lawrence Berkeley National Laboratory, all believe that the efficiency of upconversion nanoparticles should be further increased in order to increase the bioimaging quality or to improve the detection limits. Profs. Xiaogang Liu, Stefan Andersson-Engels, and Sailing He also believed that a better way of excitation is necessary to avoid heating effect and to have high quantum yield. Prof. Stefan Andersson-Engels, Prof. Xuefeng Yu and a scientist from the Molecular Foundry, Lawrence Berkeley National Laboratory (LBNL) who did not want his name to be disclosed, also believed that the low extinction of lanthanide ions strongly limits the development of upconversion nanoparticles, especially small UCNPs, for biomedical applications. Prof. Zhuang Liu and Prof Hong Zhang also had concerns about the potential long-term toxicity of UCNPs. In addition, Prof. Shukun $\mathrm{Xu}$ from Northeastern University believed that smaller sized upconversion nanoparticles, preferably less than $20 \mathrm{~nm}$, would be more relevant for in vivo applications.

What would be the next big thing in this area?

Profs. Xiaogang Liu, Stefan Andersson-Engels, Zhuang Liu, Fan Zhang, Sailing He, Hong Zhang and Xuefeng $\mathrm{Yu}$ believed that significantly increased brightness (without increasing their sizes) by either broad-band sensitizing lanthanides or a better excitation way, will impart upconversion nanoparticles a bright future. Prof. Xueyuan Chen believed that in vitro detection of tumor biomarkers, particularly those for early cancer detection, with these lanthanide-doped inorganic nano-bioprobes will be the next big thing. The scientist from LBNL also believed that efficient non-lanthanide-based upconverters will also be a breakthrough. Prof. Shukun Xu, thought that the next big thing in this field may be the successful delivery of drug molecules using UCNPs as carriers with controllable monitoring by luminescence imaging. Professor Zhuang Liu also agreed that the use of upconversion nanoparticles for some unique bio-applications that cannot be achieved by traditional fluorescent probes (e.g. QDs) can be highly significant. Prof. Jun Lin believed that the next big thing is to render these particles non-toxic and safe for administration to human beings. Prof. Xuefeng Yu men- tioned hybrid nanocomposites (e.g., plasmonic nanoparticle-based nanocomposites) and other new host materials as the next generation UCNPs. Upconversion nanoparticles are new rising stars in current biomedical fields. Nevertheless, further studies are needed to generate sufficient data to better understand the long-term toxicity and safety of the UCNPs before introducing their wide applicability in medicine.

By summarizing the original and review articles from this and the previous issue [6], as well as the opinions from the leading scientists in the area, we found numerous distinct opinions about the future directions of this emerging field. Clearly, great opportunities and challenges co-exist to advance the field of theranostic upconversion nanoparticles, with hope of eventual clinical translation for diagnosing and treating human diseases.

\section{Competing Interests}

The authors have declared that no competing interest exists.

\section{References}

1. Hao S, Chen G, Yang C. Sensing using rare-earth-doped upconversion nanoparticles. Theranostics 2013;3(5):331-345.

2. Wang C, Cheng L, Liu Z. Upconversion nanoparticles for photodynamic therapy and other cancer therapeutics. Theranostics 2013; 3(5):317-330.

3. Li X, Zhao D, Zhang F. Multifunctional upconversion-magnetic hybrid nanostructured materials: Synthesis and bioapplications. Theranostics 2013; 3(5):292-305.

4. Zhan Q, He S, Qian J, Cheng H, Cai F. Optimization of optical excitation of upconversion nanoparticles for rapid microscopy and deeper tissue imaging with higher quantum yield. Theranostics 2013;3(5):306-316.

5. Sun Y, Peng J, Feng W, Li F. Upconversion $\mathrm{NaLuF}_{4}: \mathrm{Yb}, \mathrm{Tm}$ nanophosphors for real-time upconversion luminescence imaging under ambient light and high-resolution X-ray CT imaging. Theranostics 2013; 3(5):346-353. doi:10.7150/thno.5137.

6. Chen G, Han G. Theranostic upconversion nanoparticles (I). Theranostics 2013; 3(4):289-291. doi:10.7150/thno.6382. 